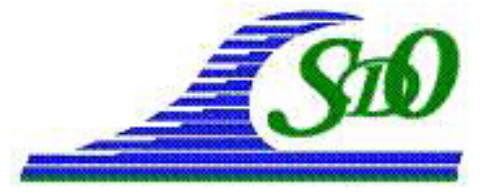

XI ${ }^{\text {èmes }}$ Journées Nationales Génie Côtier - Génie Civil

Les Sables d'Olonne, 22-25 juin 2010

DOI:10.5150/jngcgc.2010.092-B C Editions Paralia CFL

disponible en ligne - http://www.paralia.fr - available online

\title{
Bilan national du réseau de surveillance de la qualité de l'eau et des sédiments dans les ports maritimes (RÉPOM) 1997 à 2006
}

\author{
Tristan BATAILLE ${ }^{1}$, Céline LE GUYADER ${ }^{1}$, André SIMON ${ }^{1}$
}

1. CETMEF site de Brest, Département Environnement Littoral et Cours d'Eau, Technopôle Brest Iroise BP 5, 29280 Plouzané, France.

celine.le-guyader@developpement-durable.gouv.fr

\section{Résumé :}

Créé en 1997 par le Directeur de l'Eau, le RÉPOM a pour objectif le suivi national de la qualité des eaux et des sédiments dans les ports maritimes en s'appuyant sur les services chargés de la police des eaux littorales.

Après 10 ans de suivis, il est apparu nécessaire au MEEDDM d'effectuer un bilan du réseau RÉPOM, mission qui a été confiée au CETMEF. L'analyse des données acquises de 1997 à 2006 vise à déterminer quel est l'état des lieux et l'éventuelle évolution de la qualité des sédiments et des eaux de ces zones portuaires à une échelle nationale.

L'étude est décomposée en deux parties selon les deux programmes existants :

Les données du programme Sédiment qui nous renseignent sur la contamination des sédiments par plusieurs familles de substances chimiques. Nous avons cherché ici à déterminer les substances qui sont peu ou pas présentes dans les sédiments portuaires, celles qui sont présentes dans certains sites particuliers et celles qui sont largement diffusées dans les milieux portuaires. Une agrégation au niveau national a ainsi été réalisée permettant d'identifier les substances dépassant des niveaux de référence.

Les résultats de cette analyse du programme sédiment ont été cartographiés dans un cédérom interactif pour permettre une approche rapide et synthétique de la somme d'informations rassemblées sur les 10 années.

Les données du programme Eau ont quant à elles été utilisées pour mettre en évidence les sites portuaires qui présentent des niveaux élevés en sels nutritifs et/ou en microorganismes.

\section{Mots-clés :}

Génie côtier - Qualité des sédiments - Qualité des eaux - Contamination - Sédiments Ports maritimes - Dragage - Environnement littoral - Bassin hydrographique

\section{Introduction}

Les ports maritimes peuvent être soumis à des apports qui proviennent à la fois des bassins versants et des activités pratiquées dans l'enceinte du port. Chaque port est un cas particulier soumis à des flux qui sont propres à son environnement. 
Les bassins versants peuvent se caractériser par diverses activités industrielles, urbaines, agricoles pouvant conduire à des rejets directs ou à des apports diffus de nature variée, par exemple : une substance chimique liée à une activité industrielle spécifique ou des microorganismes et des sels nutritifs liés à un rejet de station d'épuration. En milieu côtier, il est encore courant que les réseaux d'eaux pluviales se rejettent dans les ports, qui sont alors le réceptacle des éventuelles pollutions provenant de l'amont.

De par leur fonction d'abri pour les navires, les ports présentent des plans d'eau calmes, propices à l'accumulation des sédiments. La plupart des matériaux dragués dans les ports et les chenaux d'accès sont riches en éléments fins, et présentent une teneur en matière organique élevée. Les sédiments fins ont tendance à accumuler les contaminants du fait de leur capacité d'adsorption et agissent comme un réservoir. Des changements chimiques au niveau des sédiments dus à la remise en suspension des sédiments via l'action des vagues, des courants ou lors d'une opération de dragage peuvent conduire à la re-mobilisation des contaminants.

En 1997, il a été décidé par le Directeur de l'Eau de mettre en place un suivi national de la qualité des eaux et des sédiments des ports maritimes, au travers d'un réseau dénommé "réseau national de surveillance de la qualité de l'eau et des sédiments dans les ports maritimes" (RÉPOM) s'appuyant sur les services chargés de la police des eaux littorales. Ceci s'est inscrit dans le contexte de la mise en œuvre alors récente des Schémas Directeurs d'Aménagement et de Gestion des Eaux (SDAGE) et de la nécessité pour les gestionnaires des milieux aquatiques, et plus particulièrement des milieux littoraux, de disposer d'information leur permettant de guider et d'évaluer leurs actions (circulaire du 7 mars 1997).

Après 10 ans de suivis, il est apparu nécessaire d'effectuer un bilan du réseau RÉPOM. L'analyse des données acquises de 1997 à 2006 cherchera à déterminer quel est l'état des lieux et l'éventuelle évolution de la qualité des sédiments et des eaux des zones portuaires à une échelle nationale à partir des données acquises.

\section{Méthodologie}

Un document de cadrage daté d'octobre 1997 est venu préciser les modalités pratiques de prélèvement des échantillons et les paramètres devant faire l'objet d'un suivi dans le cadre du RÉPOM.

Ainsi les plans d'échantillonnage du programme Sédiment ont pour but d'être représentatifs du port ou d'une partie de celui-ci. L'échantillon doit donc être représentatif d'une surface plus ou moins grande dans laquelle peuvent s'exercer des activités diverses : pêche ou plaisance par exemple. L'échantillon à analyser est ainsi constitué à partir d'échantillons élémentaires représentant toutes les situations rencontrées dans le port. Par exemple, pour un port de plaisance, il pourra s'agir de prendre en compte la plaisance permanente, le ponton visiteur, la zone d'avitaillement, la cale de mise à l'eau et l'aire de carénage. 


\section{XI ${ }^{\text {èmes }}$ Journées Nationales Génie Côtier - Génie Civil \\ Les Sables d'Olonne, 22-25 juin 2010}

Pour le programme eau, les prélèvements d'eau du réseau RÉPOM sont effectués à l'intérieur des ports ou des chenaux portuaires, afin, non pas de suivre la pollution générée par les divers rejets se déversant dans les bassins, mais de définir une valeur représentative de la qualité des eaux de ce port soumis aux diverses influences. 192 ports maritimes font ainsi l'objet d'un suivi dont 4 ports situés outre-mer. Les ports concernés se situent sur les différentes façades maritimes, présentant ainsi des situations très variées en termes de contexte économique, de bassins versants associés et de conditions environnementales. Les dragages d'entretien et d'approfondissement peuvent avoir lieu au niveau des points de prélèvements des échantillons élémentaires conduisant à introduire une possible variation dans les résultats observés pouvant être due au prélèvement effectué dans des couches sédimentaires précédemment non échantillonnées dans le cadre de ce réseau.

Afin d'appréhender toutes les activités se pratiquant dans les ports maritimes et de couvrir de façon homogène l'ensemble du littoral français, les différents types de port (commerce, pêche, plaisance, militaire) sont pris en compte et seules les principales installations sont retenues. Les ports sont classés en fonction de leur activité et de leur importance (cf. tableau 1). La périodicité des analyses est fonction de ce classement (cf. tableau 2).

\subsection{Programme Sédiment}

Pour le programme Sédiment, les paramètres devant être analysés sont :

- descriptif du sédiment : granulométrie, teneur en eau, carbone organique total et aluminium ;

- micropolluants :

- paramètres obligatoires : $\mathrm{As}, \mathrm{Cd}, \mathrm{Cu}, \mathrm{Sn}, \mathrm{Hg}, \mathrm{Pb}, \mathrm{Zn}$, hydrocarbures totaux ;

- paramètres optionnels : $\mathrm{Cr}, \mathrm{Ni}$.

D'autres paramètres peuvent également faire l'objet de mesure tels que les PCB, les HAP, le TBT et ses produits de dégradation.

Tableau 1. Activités et classes des ports maritimes pour le RÉPOM (sédiment et eau).

\begin{tabular}{|c|c|c|c|c|}
\hline \multirow[t]{2}{*}{ Classe } & \multicolumn{4}{|c|}{ Types de port } \\
\hline & Militaire & Commerce & Pêche & Plaisance \\
\hline 1 & Sans objet & $\begin{array}{l}0,2 \text { à } 0,5 \mathrm{M} \text {. tonnes / an ou } \\
50000 \text { à } 200000 \text { passagers }\end{array}$ & $500 \grave{a} 2000 \mathrm{~T} / \mathrm{an}$ & 100 à 500 anneaux \\
\hline 2 & & $\begin{array}{l}0,5 \text { à } 2 \text { M. tonnes / an ou } \\
200000 \text { à } 500000 \text { passagers }\end{array}$ & $2000 \grave{a} 5000 \mathrm{~T} / \mathrm{an}$ & 500 à 1000 anneaux \\
\hline 3 & & $\begin{array}{l}2 \grave{a} 10 \mathrm{M} \text {. tonnes / an ou } 0,5 \\
\text { à } 2 \text { M. de passagers }\end{array}$ & 5000 à $10000 \mathrm{~T} / \mathrm{an}$ & Plus de 1000 anneaux \\
\hline 4 & & $\begin{array}{l}\text { Plus de } 10 \mathrm{M} \text {. tonnes/an ou } \\
\text { plus de } 2 \mathrm{M} \text {. de passagers }\end{array}$ & Plus de $10000 \mathrm{~T} / \mathrm{an}$ & \\
\hline
\end{tabular}


Tableau 2. Fréquence de prélèvement pour les programmes Sédiment et Eau du RÉPOM en fonction de l'activité et de la classe.

\begin{tabular}{|c|c|c|c|c|c|c|c|c|}
\hline \multirow{2}{*}{$\begin{array}{l}\text { Programme } \\
\text { de suivi }\end{array}$} & \multirow[t]{2}{*}{ Type de port } & \multirow[t]{2}{*}{ Militaire } & \multicolumn{2}{|l|}{ Commerce } & \multicolumn{2}{|l|}{ Pêche } & \multicolumn{2}{|l|}{ Plaisance } \\
\hline & & & Classe 2-3 & Classe 4 & Classe 2-3 & Classe 4 & Classe 2 & Classe 3 \\
\hline SEDIMENT & $\begin{array}{l}\text { Fréquence } \\
\text { d'analyse }\end{array}$ & 1 par an & 1 sur 2 ans & 1 par an & 1 sur 3 ans & 1 par an & 1 sur 3 ans & 1 sur 2 ans \\
\hline \multirow[t]{3}{*}{$\boldsymbol{E} \boldsymbol{A} \boldsymbol{U}$} & & 4/an: tous & 4/an : tous & 4/an : tous & 4/an : tous & 4/an: tous & 2/an: & 4/an: \\
\hline & d'analyse & les 3 mois & les 3 mois & les 3 mois & les 3 mois & les 3 mois & $\begin{array}{l}1 \text { en été, } 1 \\
\text { en hiver }\end{array}$ & $\begin{array}{l}3 \text { en été, } 1 \\
\text { en hiver }\end{array}$ \\
\hline & $\begin{array}{l}\text { Nombre min. } \\
\text { de points de } \\
\text { prélèvements }\end{array}$ & 2 & 1 & 2 & 1 & 2 & 1 & 1 \\
\hline
\end{tabular}

\subsection{Programme Eau}

Pour le programme "Eau", les paramètres devant être analysés sont :

- Bactériologie : Escherichia coli ou coliformes fécaux, Streptocoques fécaux ;

- Physico-chimie :

- paramètres obligatoires : température, salinité, oxygène dissous, matière en suspension, transparence (disque de Secchi), ammonium

- paramètres optionnels : orthophosphates, nitrate, turbidité.

\section{Résultats}

\subsection{Programme Sédiment}

Pour chaque substance, sélectionnée en fonction des connaissances et de sa représentativité en matière de potentiel d'impact sur le milieu naturel dans le cas de sédiments dragués destinés à être immergés, deux seuils ont été définis correspondant à des niveaux de potentiel d'impact croissant sur un même milieu. L'appréciation effectuée s'appuie sur ces deux niveaux (N1 et N2) définissant trois classes de qualité, d'après la circulaire du 14 juin 2000. Au-dessous du niveau N1, l'impact potentiel est en principe jugé d'emblée neutre ou négligeable, les teneurs étant "normales" ou comparables au bruit de fond environnemental; entre le niveau N1 et le niveau N2, une investigation complémentaire peut s'avérer nécessaire en fonction du projet considéré et du degré de dépassement du niveau $\mathrm{N} 1$; au-delà du niveau N2, une investigation complémentaire est généralement nécessaire car des indices notables laissent présager un impact potentiel négatif de l'opération.

$\mathrm{Au}$ niveau national, l'ensemble des contaminants pour lesquels un niveau N2 existe montre un dépassement allant de $0,1 \%$ à $36,5 \%$ en fonction de la substance chimique considérée. À partir du classement en 3 classes de qualité, il est possible de 


\section{XI ${ }^{\text {èmes }}$ Journées Nationales Génie Côtier - Génie Civil Les Sables d'Olonne, 22-25 juin 2010}

hiérarchiserau niveau national les contaminants présentant des niveaux élevés au regard de la grille d'analyse utilisée.

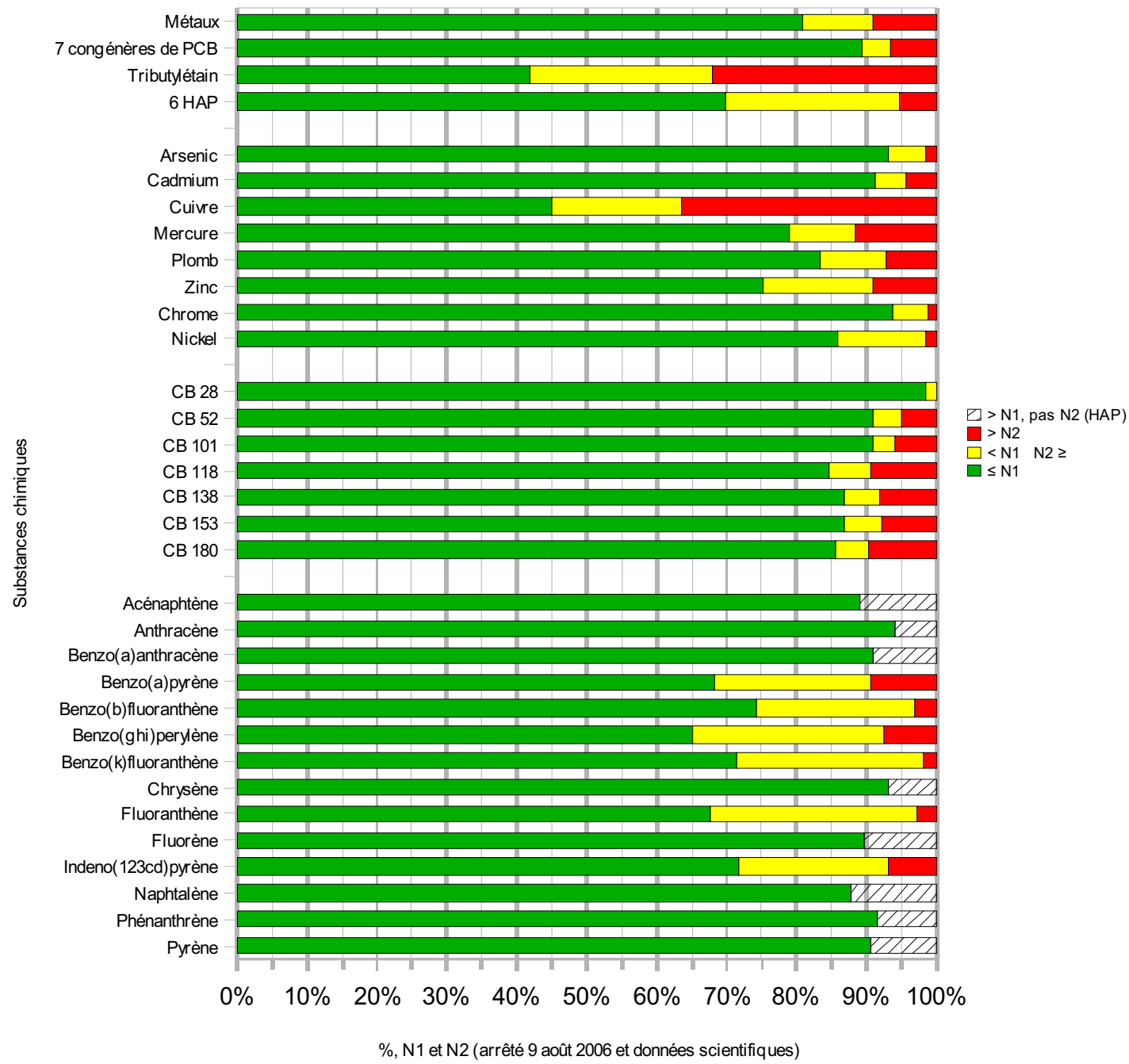

Figure 1. France, programme Sédiment du RÉPOM, 1997 à 2006, niveaux de référence.

Quatre catégories sont identifiables en fonction de leur contribution à la dégradation de la qualité des sédiments portuaires :

- TBT et Cuivre ;

- Mercure, Plomb, Zinc, PCB (CB 180, CB 153, CB 138, CB 118, CB 101), et 3 HAP

(Benzo(a)pyrène, Benzo(ghi)pérylène, Indeno(123-cd)pyrène) ;

- Cadmium;

- 3 HAP (Benzo(b)fluoranthène, Benzo(k)fluoranthène, Fluroanthène).

Pour chaque bassin hydrographique, un graphique positionne de la même manière qu'à l'échelle nationale les données pour les contaminants chimiques mesurés dans les sédiments par rapport aux niveaux de référence $(\mathrm{N} 1, \mathrm{~N} 2)$. 
Dans le cas par exemple du bassin hydrographique "Loire-Bretagne", les contaminants qui ressortent pour leur dépassement du seuil N2 sont :

- TBT, Cuivre ;

- 6 HAP ;

- PCB (excepté le CB 28);

- Zinc.

Enfin, deux représentations sont possibles pour exprimer la contamination des sédiments par une substance. L'une au format agrégé comme pour l'échelon national et par bassin hydrographique, et l'autre sous forme de cartographie interactive comme représenté sur la figure 2, qui permet l'analyse de l'évolution des contaminants dans les sédiments portuaires pour les années allant de 1997 à 2006. Chaque paramètre qui se distingue (par un dépassement de seuil par exemple) fait l'objet d'une analyse plus approfondie sur les origines possibles, parfois historiques.

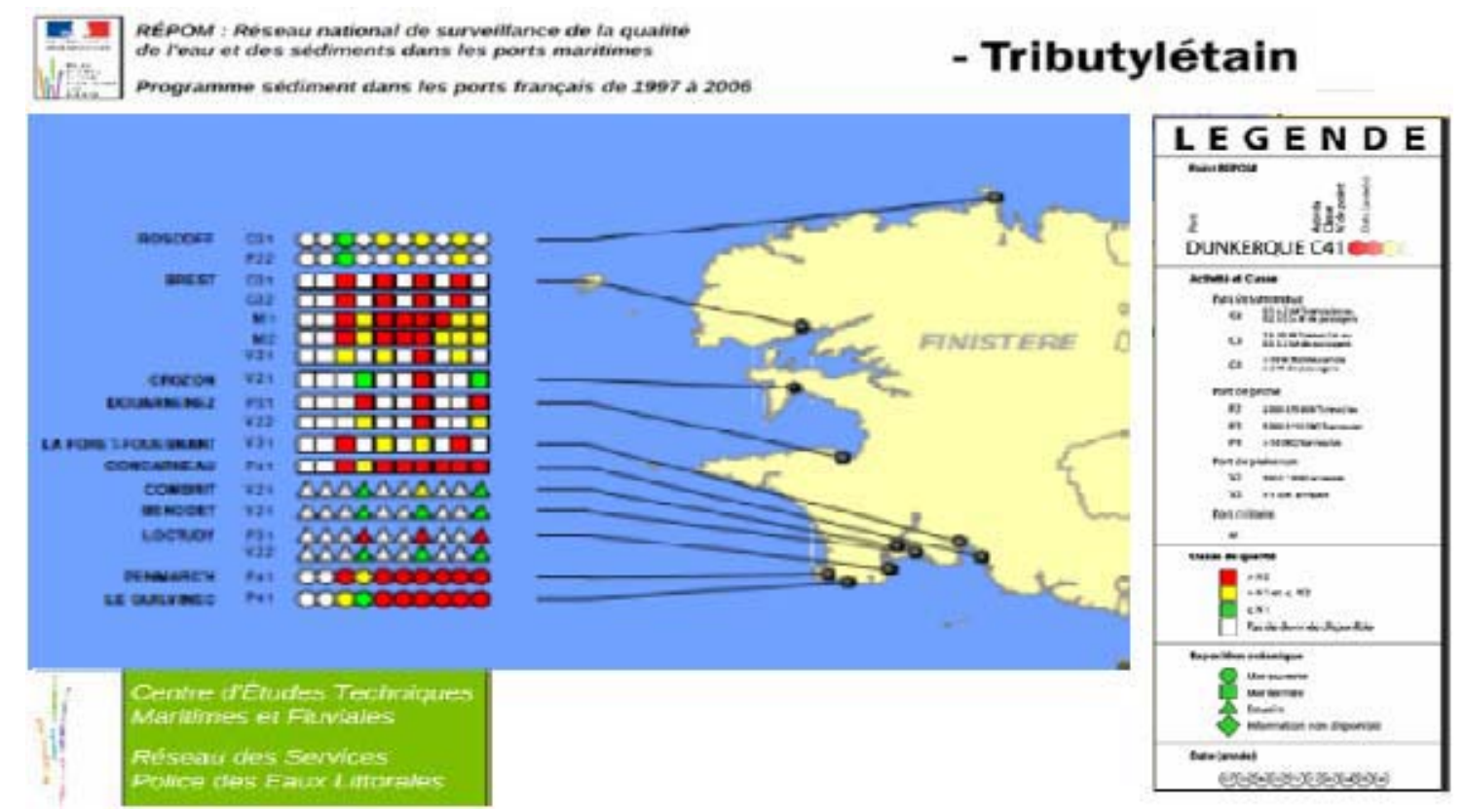

Figure 2. Représentation cartographique de la contamination des sédiments portuaires au tributylétain de 1997 à 2006 - zoom sur le département du Finistère.

En ce qui concerne l'exemple du tributylétain, au niveau national, près d'une donnée sur trois dépasse N2. Même si l'utilisation du TBT a été réduite aux navires de plus de 25 mètres au début des années 80 , il est fortement associé aux sédiments marins du fait de son caractère persistant. Environ un quart des ports suivis disposant de données (27\%) ne présentent aucun dépassement de N1. L'ensemble des bassins hydrographiques est concerné par des dépassements de N2. Au niveau national, l'Artois-Picardie est le secteur le moins touché, avec seulement 1 dépassement de N2 observé sur 83 données acquises en 10 ans de suivi. Ces résultats dans le bassin Artois-Picardie s'expliquent en 


\section{XI $I^{\text {èes }}$ Journées Nationales Génie Côtier - Génie Civil}

Les Sables d'Olonne, 22-25 juin 2010

partie par le positionnement des stations de mesures qui ne sont pas localisées à proximité immédiate des secteurs de réparation navale.

\subsection{Programme Eau}

Les données obtenues dans l'eau des ports sont analysées par port et agrégées sur 10 ans. Chaque port sera représenté par une valeur et les ports sont comparés entre eux. Chaque port est décrit à partir du centile 90 qui permet d'éliminer quelques valeurs extrêmes qui pourraient être attribuables à des évènements ponctuels non représentatifs, tout en mettant en avant les valeurs hautes de l'échantillon de données. Le centile 90 est la valeur pour laquelle $90 \%$ des données sont inférieures et $10 \%$ supérieures. À raison de 4 mesures par an sur 10 ans, décrire l'échantillon à partir de ce paramètre consiste à ne pas considérer 4 événements extrêmes.

Si l'on prend l'exemple d'Escherichia Coli, le 90e centile est calculé pour 150 ports. Plusieurs sites portuaires ressortent alors au niveau national. Ainsi 12 ports présentent un 90e centile supérieur à 10000 UFC / 100 mL. Il s'agit de Boulogne-sur-mer pêche, Port-en-Bessin pêche, Saint-Brieuc commerce et plaisance, Les-Sables-d'Olonne plaisance, Bordeaux commerce, Saint-Jean-De-Luz plaisance, Hendaye pêche, Sète pêche, Marseille Bassin Est commerce, Ajaccio commerce et plaisance. Il y a 4 sites sur 37 ports de pêche (11\%), 4 sites sur 26 ports de commerce (15\%) et 4 sites sur 86 ports de plaisance $(5 \%)$.

\section{Conclusion}

L'analyse des données du RÉPOM Sédiment après 10 ans de suivi, met en évidence une contamination importante des sédiments portuaires par deux polluants, le tributylétain et le cuivre dont les sources principales sont la diffusion provenant des peintures antisalissures des navires et les rejets liés aux activités de carénage et de réparation navale. L'arsenic n'est globalement pas retrouvé dans ces sédiments portuaires. Le nickel et le chrome ne sont pas observés à des niveaux élevés à part pour quelques ports. Pour le programme Eau du RÉPOM, les paramètres retenus (et notamment les microorganismes d'origine fécale) pour cette étude nationale font ressortir 12 sites, sur 150 ports dont la qualité des eaux a été évaluée, sans lien apparent avec l'activité. La mesure des éléments nutritifs met en évidence qu'à l'échelle nationale la majorité des ports suivis dans le cadre du RÉPOM ne présente pas de concentrations élevées en éléments nutritifs dans l'eau. Seuls cinq sites présentent des niveaux qui seraient importants pour les nutriments.

Les sources de microorganismes et d'éléments nutritifs sont très variées, les rejets peuvent provenir d'eaux usées domestiques, de rejets urbains, agricoles ou industriels. Trois sites ressortent à la fois pour leurs teneurs élevées en éléments nutritifs et en microorganismes, il s'agit de Saint-Brieuc (commerce), Les Sables d'Olonne (plaisance) et Ajaccio (commerce). 


\section{Remerciements}

A l'ensemble des Services Police des Eaux Littorales qui ont ainsi permis le recensement exhaustif des données du réseau et en particulier aux services suivants qui ont rendus possible l'analyse plus fine de l'évolution des contaminants portuaires :

Eric Pain /SPEL50 ; Arnaud Rouilly/SPEL44 ; René Soulard/SPEL85 ; Marie-Christine Bertrandy/SPEL13 ; Agnès Rosso-Darmet/SPEL83

Remerciements également à l'ensemble des membres du COPIL et au bureau des milieux marins de la DGALN qui permettent de fixer les orientations du réseau REPOM ainsi que son évolution pour les années à venir, afin de prendre en compte au mieux les problématiques du ministère (DCE) et européennes (OSPAR notamment).

\section{Références bibliographiques}

ABARNOU A., LE GUELLEC A-M., LOIZEAU V. (2002). Les polychlorobiphényles (PCB) dans l'environnement littoral, dans Bulletin RNO 2002, pp 13-24.

ALZIEU C., MICHEL P., CHIFFOLEAU J.-P., BOUTIER B., ABARNOU A. (1999). Chapitre $V$ : Contamination chimique des sédiments. Dans Dragages et environnement marin - Etat des connaissances, coordinateur Claude Alzieu, Editions IFREMER, pp 70-110.

EGGLETON J., THOMAS K. (2004). A review of factors affecting the release and bioavailability of contaminants during sediment disturbance events, Environment International, vol. 30, pp 973-980. doi:10.1016/j.envint.2004.03.001

GUILlAUD J-F., AMINOT A. (1990). Devenir des éléments nutritifs en zone littorales, dans La Mer et ses Rejets Urbains, Actes de colloques, IFREMER, pp 27-34. MERCERON M., LE BOZEC G. (1999). Motivations et présentation du programme «Bassins versants et transmission des pollutions au littoral» dans Pollutions diffuses $d u$ bassin versant au littoral, Actes de colloques, IFREMER, pp 7-12.

QUINIOU F. (2004). Impact des dragages sur l'environnement littoral de la prévision au suivi dans Thème D Dragages et valorisation des produits de dragage : quelles recherches complémentaires ?, Journées Scientifiques et Techniques du CETMEF, Actes de colloques, pp 259-264. 